\title{
Analysis of 1 Million 3D Foot Scans from North America, Europe, Asia and Australia
}

\author{
Ales JURCA ${ }^{* 1,2}$, Jure ZABKAR ${ }^{3}$, Saso DZEROSKI ${ }^{4,2}$ \\ ${ }^{1}$ Volumental $A B$, Stockholm, Sweden; \\ 2 Jožef Stefan International Postgraduate School, Ljubljana, Slovenia; \\ ${ }^{2}$ Faculty of Computer and Information Science, University of Ljubljana, Slovenia; \\ ${ }^{3}$ Jozef Stefan Institute, Ljubljana, Slovenia \\ DOI: $10.15221 / 18.281 \quad$ http://dx.doi.org/10.15221/18.281
}

\begin{abstract}
The purpose of this study was to perform a preliminary analysis of 1 million 3D Foot scans. Data were collected with Volumental 3D foot scanners, located in footwear stores in North America, Europe, Asia and Australia. Foot length and foot width measurements were extracted from the 3D scans using an algorithmic method. Kernel density estimation was performed for each gender and region to compare the distributions of foot length and relative foot width across the studied regions. The results show that Asian customers have significantly shorter and relatively wider feet than customers in other regions.
\end{abstract}

Keywords: 3d foot scanning, foot scan database, foot dimensions, gender differences, ethnic differences

\section{Introduction}

Proper fit is one of the most important features of footwear when customers are purchasing shoes. A shoe last is the primary determinant of the inner shape of a shoe. This is a wooden, plastic or metal object with a shape similar to that of a human foot, used to form a shoe during the shoe manufacturing process. Actual foot measurements are important input data for designing shoe lasts so that shoes will have a higher likelihood of fitting a customer's foot [1,2]. With the latest developments in 3D foot scanning technology, foot measurements have become more accurate, repeatable and easier to acquire as compared to manual foot measurements. Previous studies have analyzed 3D foot measurements on small datasets, limited to smaller geographical regions $[3,4,5,6]$.

Recent penetration of 3D foot scanning technology into footwear retail enables the possibility to build large databases of 3D scans of customers' feet from various geographical locations. These databases of feet provide new opportunities for research of foot dimensions and shapes.

Volumental $A B$ in Sweden have developed a fast, accurate and user-friendly 3D foot scanning solution, providing an outstanding user experience for shoe shoppers. Sales associates are using scan results to better accommodate customers' needs. Foot scanners are currently located in more than 1000 footwear stores in 35 countries worldwide. The objective of this study was to perform a distribution analysis of foot length and relative width measurements extracted from 3D foot scans. The study was performed on 3D foot scans acquired in 4 large regions: North America, Europe, Asia and Australia. To our knowledge, this is the first known anthropometric study analyzing such a large number of 3D foot scans from various continents.

\section{Methodology}

\subsection{D foot scanner}

Volumental 3D foot scanners were first used to scan customers' feet (figure 1). The scanner consists of a base platform and four camera towers. A customer needs to first take off their shoes before scanning. They can leave their socks on or go barefoot. Socks of any color or material can be worn by the customer during scanning. The customer stands on the base platform with both feet positioned slightly apart from each other. Body weight should be equally distributed between both feet. The scanning process takes 5 seconds.

Four depth cameras are used to capture the 3D shape of the feet. The cameras are positioned so that both feet are scanned simultaneously. They capture the visible surfaces of both feet which are visible from each camera position. The results of the camera captures create four point clouds of parts of feet surfaces.

*ales.jurca@volumental.com; +46 7224550 29; www.volumental.com 
Figure 2 shows an example of a point cloud captured by the front-right camera. A post-processing algorithm combines the point clouds into one point cloud for each foot, assuming the plantar part of the foot (touching the base platform while being scanned) is flat. In the last post-processing step, a mesh file for each foot is created.

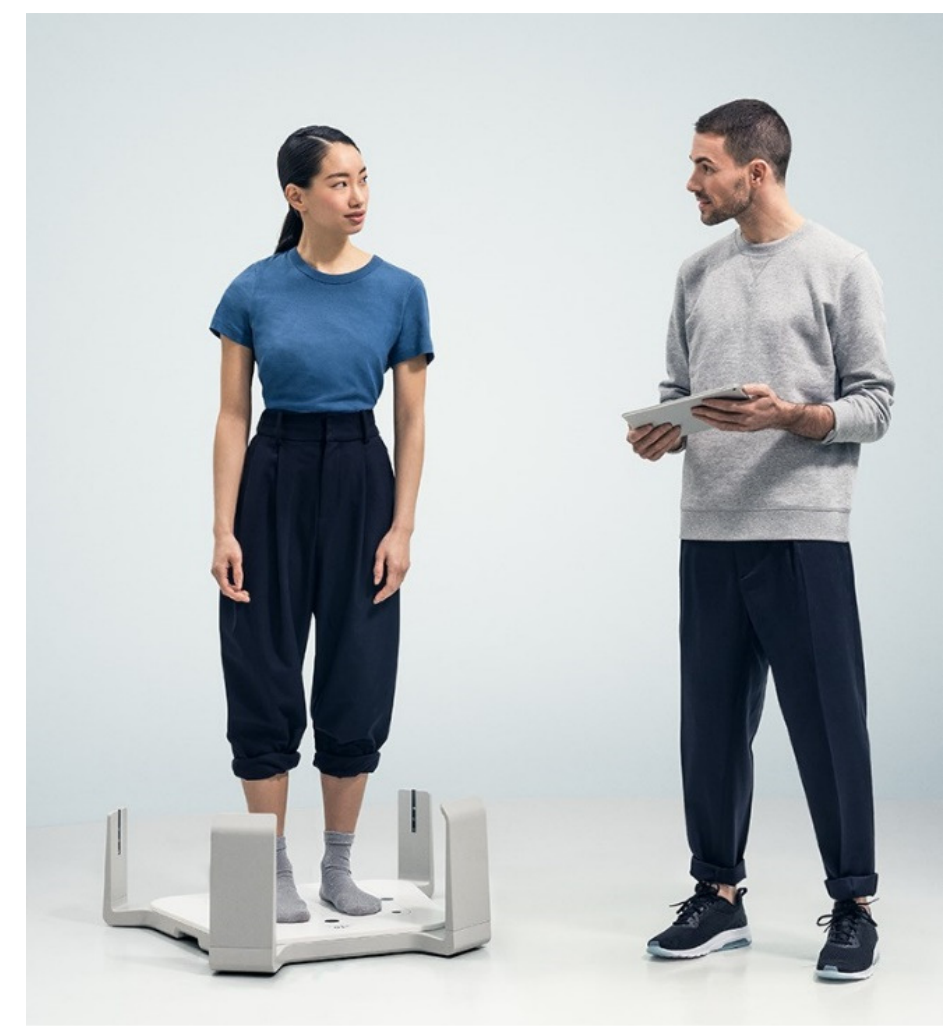

Fig. 1. Volumental 3D foot scanner

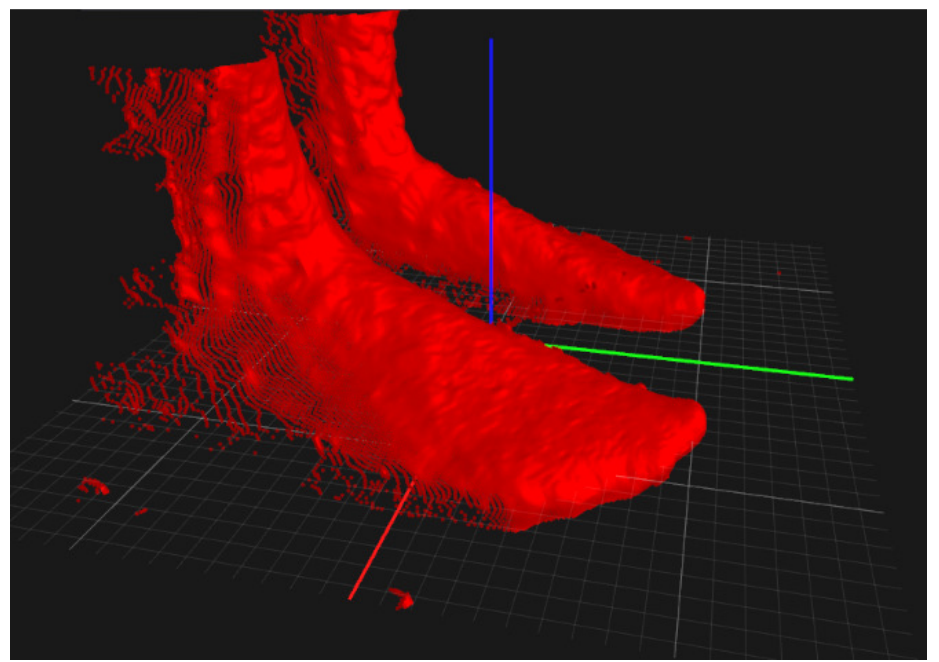

Fig. 2. Unfiltered/raw point cloud captured by front right depth camera

\subsection{Graphical User Interface}

A sales associate uses a tablet to interact with the scanner during the scanning process and to show the scan results to the customer. The scanner and the tablet are connected via a Wi-Fi hotspot located in the base platform of the scanner. In the first step of the scanning process, the sales associate selects gender and starts a scan. After the scan is successfully processed, 3D meshes and foot measurements of both feet are displayed (figure 3). The customer can create an account to store the scan for later use. Best fitting styles are recommended to the customer based on the foot scan data and inventory data of the store. 


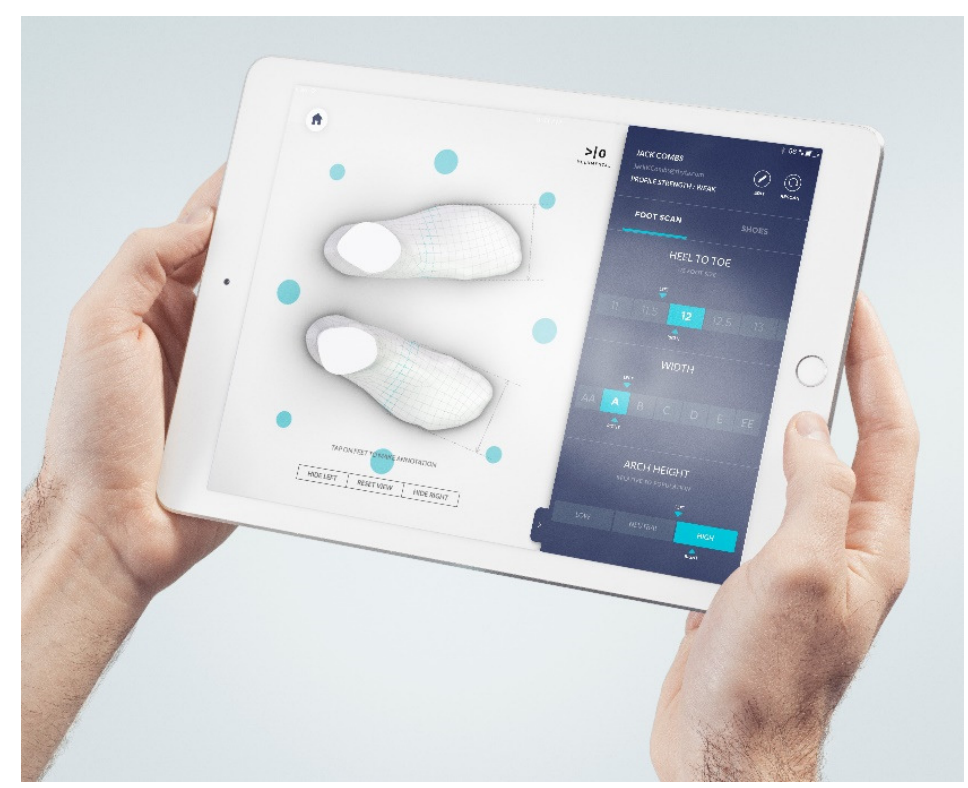

Fig. 3. Scan results

\subsection{Collection of 3D foot scan data}

The foot scan data comprises 1,030,408 customers, which have been scanned between November $1^{\text {st }}$ 2017 and August $31^{\text {st }} 2018$ by some of the footwear brands and retailers that are using the Volumental foot scanning solution in footwear retail, namely: Bauer Hockey, Ecco, Fleet Feet, Lotte, New Balance, Road Runner Sports and Wintersteiger. Scanners are located in four global regions: North America, Europe, Asia and Australia. All scanners are connected to the internet, which enables automatic transfer of 3D mesh files to a cloud server where the foot mesh files are stored and further analyzed.

\subsection{Analysis of foot measurements}

Foot length and width measurements were extracted from each 3D mesh using methods described in [3] and presented in figure 4.
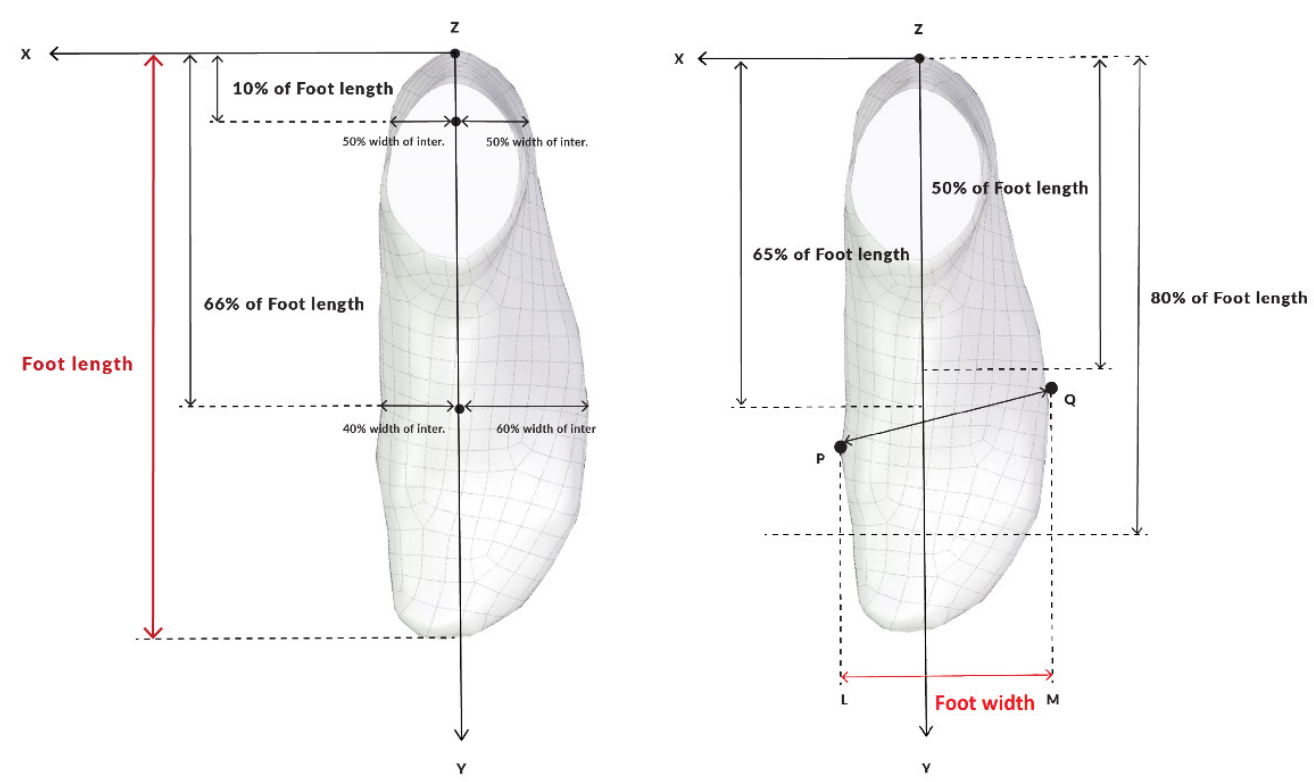

Fig. 4. Extraction of foot length and width from a $3 D$ mesh

Relative foot width was calculated by equation 1 .

$$
\text { relative_foot_width }=\frac{\text { foot_width }}{\text { foot_length }}
$$


The Pandas, Matplotlib and Seaborn python libraries were used for analysis of the collected foot measurements and visualization of the results. Kernel density estimation (KDE) was performed for each gender and region to compare the distributions of foot length and relative foot width across the studied regions.

\section{Preliminary results and discussion}

\subsection{Distribution of scans per gender and region}

Both genders were well represented in the dataset (figure 5).

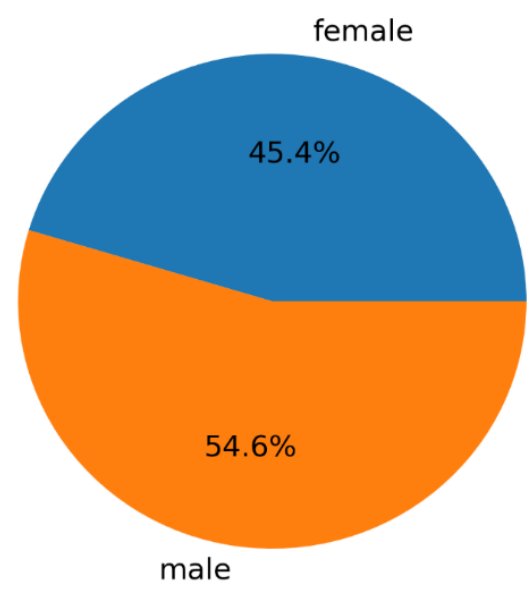

Fig. 5. Distribution of scans per gender

Figure 6 shows that the majority of scans were made in North America. Even though there were a smaller percentage of scans from Asia and Australia, the absolute numbers were large enough to include these two regions in the analysis. There were 21,532 male and 14,768 female customers scanned in the Asia region. The number of scans in Australia was 2,237 for male and 1,673 for female customers, which is still higher than most of the analyses of foot scans done in previous studies.

Distribution of scans per region - male

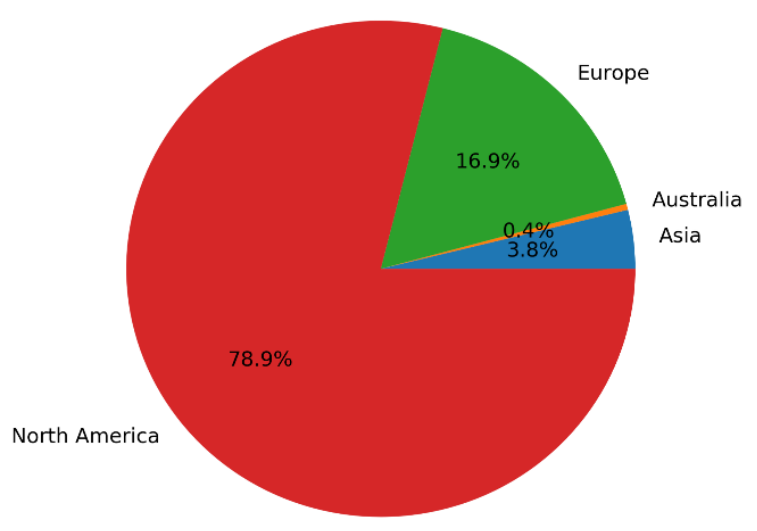

Distribution of scans per region - female

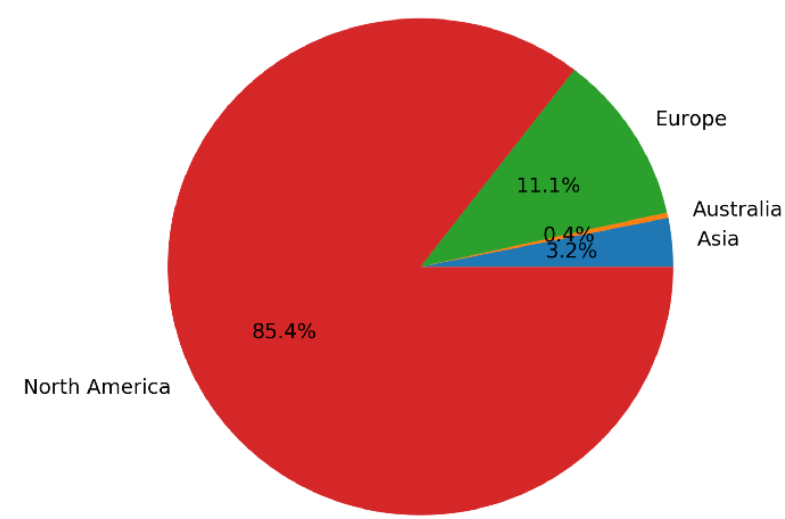

Fig. 6. Distribution of scans per region

\subsection{Foot length distribution}

Figure 7 shows the kernel density estimate (KDE) of foot length distribution for male and female customers. For male customers, there is a significant difference between the distribution (KDE) for Asia and other regions. The difference shows that Asian customers have significantly shorter feet than customers in other regions. 
Having two peaks, the KDE for Australia is slightly different than the KDE for Europe and North America. The right peak of the KDE for Australia is aligned with the peaks of the KDEs for Europe and North America, while the left peak is closer to the peak of the KDE for Asia. While the reason for the left peak on the KDE for Australia is not completely clear, one explanation could be the Asian population living in Australia.

Very similar conclusions can be drawn from the KDEs for female customers.

A comparison of the male and female KDEs shows significant differences between male and female customers. Obviously, male customers have longer feet than female customers in all four regions.
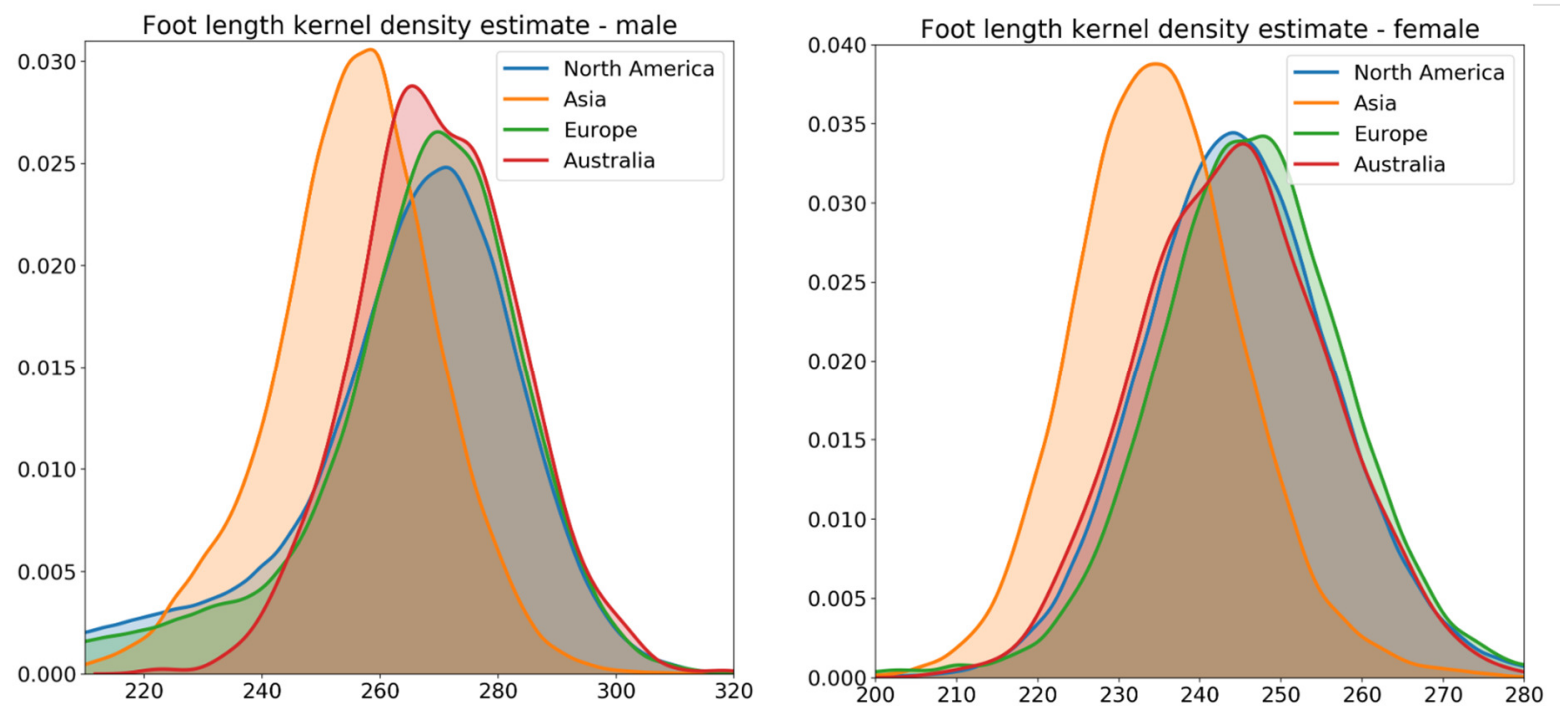

Fig. 7. Foot length kernel density estimate

\subsection{Relative foot width distribution}

Figure 8 shows the kernel density estimate (KDE) of relative foot width distribution for male and female customers. Both charts show significant differences between Asian customers and customers from other regions. Male and female Asian customers have relatively wider feet than other customers.
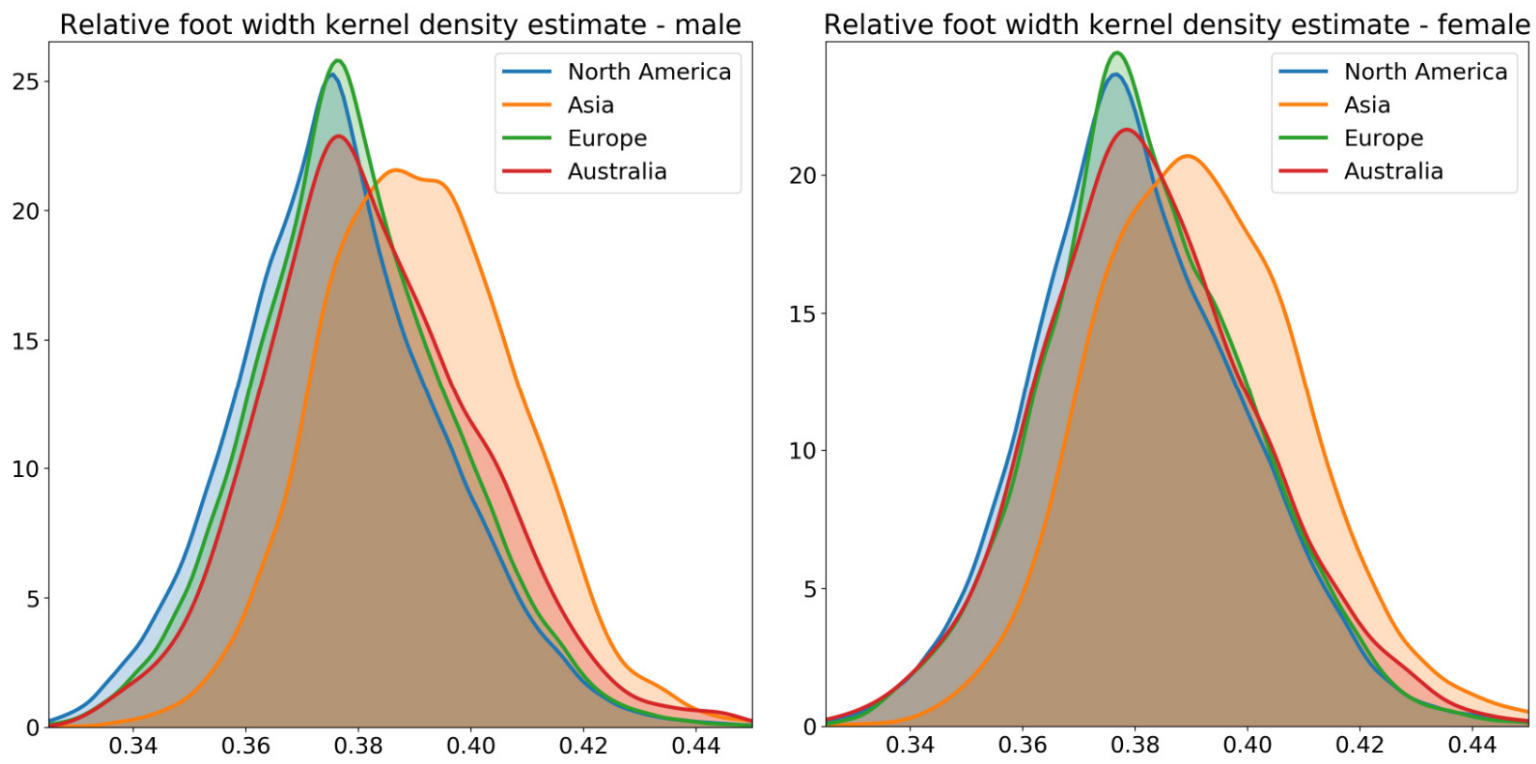

Fig. 8. Relative foot width kernel density estimate 


\section{Conclusions and future work}

The aim of this study was to perform a preliminary analysis of the 3D foot scan data from four regions. The results showed significant differences between the foot scans from Asia and other regions for both analyzed foot measurements. The foot length distributions are very useful for footwear brands in deciding about the size range of shoe styles, depending on the target market. Footwear retailers can use these findings when planning inventory per size. The relative foot width distributions should be considered when designing the shoe lasts so that shoes have a higher likelihood of fitting customers' feet of the target market.

The analysis of the presented 3D foot scan database is still in early stages. Additional data analysis and descriptive statistic methods will be used to quantitatively describe foot measurements and compare the results between the genders and the regions. The foot scans in Europe and Asia are collected in many countries, therefore comparisons between countries could provide additional insights into foot dimensions.

Hundreds of thousands of scans are currently added to the Volumental 3D foot database every month. Furthermore, additional foot scanners will be installed in footwear stores in all four regions. Additional scans will increase the sample sizes of the different countries within the regions and improve comparisons of the foot measurements between the countries.

\section{References}

[1] F-T. Cheng et. al, "A systematic approach for developing a foot size information system for shoe last design", in International Journal of Industrial Ergonomics, Volume 25, Issue 2, February 2000, pp.171-185, https://doi.org/10.1016/S0169-8141(98)00098-5

[2] A. Luximon and Y. Luximon, "Shoe-last design innovation for better shoe fitting", in Computers in Industry, Volume 60, Issue 8, October 2009, pp. 621-628, https://doi.org/10.1016/j.compind.2009.05.015

[3] A. Jurca et. al, "DOROTHY Mass Foot Measurement Campaign", in Proc. of $1^{\text {st }}$ International Conference on 3D Body Scanning Technologies, Lugano, Switzerland, 19-20, 2010, pp.338-344, http://dx.doi.org/10.15221/10.338.

[4] I. Kraus et. al, "Sex-related differences in foot shape of adult Caucasians - a follow-up study focusing on long and short feet", in Ergonomics, Vol.54, No.3, 2011, pp.294-300, https://doi.org/10.1080/00140139.2010.547605

[5] Y-C. Lee and M-J. Wang, "Taiwanese adult foot shape classification using 3D scanning data", in Ergonomics, Vol.58, No.3, pp.513-523, 2015, https://doi.org/10.1080/00140139.2014.974683

[6] J.W. Wannop et. al, "Development of a Footwear Sizing System in the National Football League", in Sports Health, 2018, https://doi.org/10.1177/1941738118789402 